\title{
Cannulation of Obscured Papilla Aided By Using a Balloon to Flatten Out Duodenal Folds, in a Patient with Acute Pancreatitis
}

Cannulation of the papilla is the indispensable step in performing endoscopic retrograde cholangiopancreatography (ERCP) [1], but sometimes there are technical problems that can lead to failure [2]. In patients with gallstone pancreatitis, there is a potential difficulty for endoscopists due to the possible presence of duodenal edema which may hide the papilla of Vater [3]. We describe here a case of difficult cannulation, achieved by using a balloon to reduce the duodenal folds.

A 78-year-old man was referred to the emergency room of our hospital for hyperemesis and syncope. Blood tests revealed a slight increase in bilirubin $(1.82 \mathrm{mg} / \mathrm{dl})$, elevation of the pancreatic enzymes (amylase $1001 \mathrm{U} / \mathrm{l}$, lipase 1647 $\mathrm{U} / \mathrm{l}$ ), slight increases in transaminases, alkaline phosphatase (ALP) and gammaglutamyl transpeptidase (GGTP), and a normal calcemia level.

The symptoms worsened 4 days later, with increased abdominal pain referred to the back, and the persistence of nausea and hyperemesis. We repeated a biochemical evaluation, which showed an increase of amylase (1417 U/L) and bilirubin $(3.52 \mathrm{mg} / \mathrm{dl})$ associated with a decrease in calcium $(5.8 \mathrm{mg} / \mathrm{dl})$. The patient had an abdominal computed tomography (CT) scan, and the radiologist identified the typical features of severe pancreatitis (hypodensity and enlargement of the pancreas with intraparenchymal and peripancreatic fluid collection).

We decided to perform ERCP, to remove the stones from the biliary tree via sphincterotomy. When we reached the duodenum, we found edema associated with enlargement of the duodenal folds, which did not allow us to see the papilla (Figure 1).

An inflated balloon for stone extraction (Extractor XL 5147; Boston Scientific Microvasive, Nanterre, France) was used to flatten the folds, because they were too stiff to be moved or pulled aside in a sys-

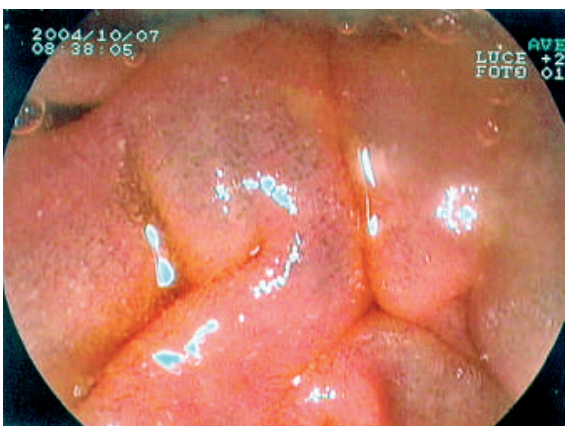

Figure 1 The papilla of Vater is obscured by the edematous duodenal folds.

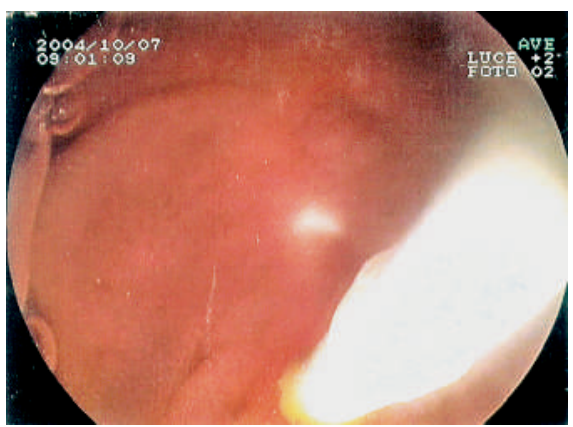

Figure 2 Cannulation of the papilla after the duodenal fold has been flattened somewhat with a balloon catheter (balloon inflated).

tematic search for the papilla with the sphincterotome, as we normally do in this kind of situation. After a few attempts, randomly inflating the balloon against the wall in different duodenal areas, we succeeded in finding the papilla. We then inserted a guide wire through the balloon catheter and cannulated the papilla without changing the instrument (Figure 2).

Finally, we used the sphincterotome on the guide wire to carry out endoscopic sphincterotomy, and then removed the stones from the biliary tree using the same balloon employed for cannulation. The patient's clinical picture improved rapidly, with a full recovery after a short period of hospitalization.

This case illustrates an ingenious way for the endoscopist to reach the papilla in the case of duodenal edema which ren- ders other methods impractical, performed using a universally available device, the balloon catheter. However, this cannot be regarded as a definitive solution to the problem because a certain amount of luck is required in addition to the endoscopist's skilfulness.

Competing interests: Not declared

V. Cennamo, D. Baroncini, C. Fabbri, F. Ferrara, S. Guersi, N. D'Imperio Division of Gastroenterology, BellariaMaggiore Hospital, Bologna, Italy

\section{References}

${ }^{1}$ Cotton PB. Cannulation of the papilla of Vater by endoscopy and retrograde cholangiopancreatography (ERCP). Gut 1972; 13: 1014- 1025

${ }^{2}$ Carr-Locke DL. Biliary access during endoscopic retrograde cholangiopancreatography. Can J Gastroenterol 2004; 18: 251-254

${ }^{3}$ Martin DF, Tweedle D, Haboubi NY. Clinical practice of ERCP. London: Churchill Livingstone; 1998: 85 - 100

\section{Corresponding Author}

\section{Cennamo, M.D.}

Division of Gastroenterology

Bellaria Hospital, Via Altura 3

40139 Bologna, Italy

Fax: $\quad$ +39-051-6225247

E-mail: vincenzo.cennamo@ausl.bo.it 